Asia Pac. J. Math. 2020 7:9

ASIA PACIFIC ACADEMIC

\title{
A NUMERICAL APPROXIMATION TO THE UNIQUE SOLUTION OF KDVB EQUATION UNDER THE PRABHAKAR FUNCTION
}

\section{MOHAMMADHOSSEIN DERAKHSHAN, AZIM AMINATAEI*}

Faculty of Mathematics, K. N. Toosi University of Technology, P. O. Box: 1676-53381, Tehran, Iran

*Corresponding author: ataei@kntu.ac.ir

Received Mar. 18, 2020

АвSTRACt. In this paper, we consider the fractional Korteweg-de Vries-Burgers (KdVB) equation as follows:

$$
{ }^{C} \mathbb{D}_{\rho, \mu, \omega}^{\gamma} u(x, t)=\sigma \frac{\partial^{2} u(t, x)}{\partial x^{2}}-2 u \frac{\partial u(t, x)}{\partial x}-\delta \frac{\partial^{3} u(t, x)}{\partial x^{3}}, 0<\mu \leq 1,
$$

where the operator ${ }^{C} \mathbb{D}_{\rho, \mu, \omega}^{\gamma}$ is a Caputo-Prabhakar fractional derivative of order $\mu$. We obtain the existence and uniqueness of the solutions of this kind of fractional differential equation by using compositions of the fractional integration operator with the fractional derivative operator, some sufficient conditions. Also, we are set up to simulate the unique solutions of the KdVB by using the numerical approximation that this approximation is based on the two-step Lagrange polynomial interpolation method. Finally, the numerical experiments are carried out to support the theoretical claims.

2010 Mathematics Subject Classification. 26A33, 34k20, 44A10.

Key words and phrases. Prabhakar function; KdVB-equation; fractional derivative; Lagrange polynomial interpolation.

\section{InTRODUCTION}

In the last decades, non-integer orders of differentiation and integration and fractional differential systems have an important role in fractional calculus $[2-5,8-10,14]$. Also, due to its application in engineering sciences and mathematical fields such as probability and the study of stochastic processes attention many writers are located. A KdVB equation is defined

DOI: 10.28924/APJM/7-9 
as follows [7]:

$$
\frac{\partial u(t, x)}{\partial t}=\sigma \frac{\partial^{2} u(t, x)}{\partial x^{2}}-2 u \frac{\partial u(t, x)}{\partial x}-\delta \frac{\partial^{3} u(t, x)}{\partial x^{3}}
$$

where $\sigma$ and $\delta$ are the two perturbation parameters and when $\sigma=0$, the relation (1-1) becomes Korteweg-de Vries (KdV) equation, also when $\delta=0$, the relation (1-1) corresponds to the Burgers equation. Hence, the KdVB equation are a type of combination of both Burgers and Korteweg-de Vries equations. The KdVB equation was first introduced by Su \& Gardner (1969) [16]. The equation (1-1) considered as appropriate system for a wide range of nonlinear systems in the weak nonlinearity and long wavelength approximations, since it contains both damping and dispersion. The two states can be considered for the solutions of the differential equation as follows [7]:

1) the steady state solutions of the KdVB equation are monotonic shocks, when diffusion dominates dispersion,

2) the steady state solutions of the KdVB equation are oscillatory shocks, when dispersion dominates diffusion.

These equations play an important role in the field of wave propagation through liquid filled elastic tubes, shallow water waves on a viscous fluid [15], current flow in electrical flow and pulse in biological chains. The numerical approximations results of fractional differential systems have been the main goal in contributions. In [6] used the Ritz approximation for numerical solutions of fractional partial differential equations (FPDEs)in the sense of the Caputo fractional derivative to develop a scheme for solving the Burger's equation. Artstein [1] is implement the dispersive approximation scheme of Goodman and Lax [12] for solving the KdVB equation. Soliman [17] used a variational iteration method for finding the wave solution of the Lax's seventh-order KdV (for short, LsKdV) and Korteweg-de Vries Burgers (for short, KdVB)equations. In the end Goufo [7] are using the two-step Lagrange polynomial interpolation method for solving the Korteweg-de Vries-Burgers equation and in this paper, there is a study of the existence and uniqueness of this kind of equation. In this paper, we are going to by replacing $\frac{\partial u(t, x)}{\partial t}$ with ${ }^{C} \mathbb{D}_{\rho, \mu, \omega}^{\gamma}$ in the definition of $\mathrm{KdVB}$ equation (1-1) and discuss the existence, uniqueness and some numerical simulations approximating of the solutions of this fractional equation. Here we introduce a fractional Korteweg-de Vries-Burgers in the sense of Caputo-Prabhakar fractional derivative of order $\mu$ as follows:

$$
{ }^{C} \mathbb{D}_{\rho, \mu, \omega}^{\gamma} u(x, t)-\sigma \frac{\partial^{2} u(t, x)}{\partial x^{2}}+2 u \frac{\partial u(t, x)}{\partial x}+\delta \frac{\partial^{3} u(t, x)}{\partial x^{3}}=0,0<\mu \leq 1,
$$


such that this kind of derivative ${ }^{C} \mathbb{D}_{\rho, \mu, \omega}^{\gamma}$ is a novel generalization of derivatives of both RiemannLiouville and Caputo types. The Caputo-Prabhakar fractional derivative is obtained by modifying the Caputo derivative by extending its kernel with a three-parameter Mittag-Leffler function that known as Prabhakar function and is defined as follows:

$$
E_{\rho, \mu}^{\gamma}(z)=\sum_{n=0}^{\infty} \frac{(\gamma)_{n}}{n ! \Gamma(\rho n+\mu)} z^{n}, \Re(\rho), \Re(\mu)>0, \gamma>0, z \in \mathbb{C}
$$

where $\Gamma($.$) denotes the Euleŕs gamma function and also this prabhakar function, a function$ which extends the well-known two-parameter Mittag-Leffler function and this function are a lot of applications in anomalous dielectrics of Havriliak-Negami, stochastic processes, some fractional boundary-value problems, dynamical models of spherical stellar systems. For this purpose, in Section 2 we recall the fundamental definitions in fractional calculus and in this section introduces the fractional derivative with Prabhakar Kernel. In Section 3, we survey the existence, uniqueness and the numerical approximation scheme based on the two-step Lagrange polynomial interpolation for KdVB-equation with Caputo-Prabhakar fractional derivative, also in this section the simulation results are discussed.

\section{Preliminaries}

In this section, we recall some definitions and lemmas of fractional integral and differential operators which are used in the next sections.

Definition 2.1. $[13,18]$. For $0<\alpha \leq 1$ and $f \in L^{1}[a, b], 0<t<b \leq \infty$, the left-sided and the right-sided Riemann-Liouville fractional integrals and derivatives of order $\alpha$ are defined as

$$
\begin{aligned}
& I_{a^{+}}^{\alpha} f(t)=\frac{1}{\Gamma(\alpha)} \int_{a}^{t} f(\tau)(t-\tau)^{\alpha-1} d \tau \\
& I_{b^{-}}^{\alpha} f(t)=\frac{1}{\Gamma(\alpha)} \int_{t}^{b} f(\tau)(\tau-t)^{\alpha-1} d \tau
\end{aligned}
$$

$$
\begin{aligned}
& D_{a^{+}}^{\alpha} f(t)=\frac{1}{\Gamma(\alpha)} \frac{d}{d t} \int_{a}^{t} f(\tau)(t-\tau)^{-\alpha} d \tau, \\
& D_{b^{-}}^{\alpha} f(t)=-\frac{1}{\Gamma(\alpha)} \frac{d}{d t} \int_{t}^{b} f(\tau)(\tau-t)^{-\alpha} d \tau .
\end{aligned}
$$


Also, for the absolutely continuous function $f$, the left-sided and the right-sided Caputo fractional derivatives of order $\alpha$ are defined as follows

$$
\begin{aligned}
& { }^{C} D_{a^{+}}^{\alpha} f(t)=I_{a^{+}}^{1-\alpha} \frac{d}{d t} f(t)=\frac{1}{\Gamma(1-\alpha)} \int_{a}^{t}(t-\tau)^{-\alpha} \frac{d}{d \tau} f(\tau) d \tau, \\
& { }^{C} D_{b^{-}}^{\alpha} f(t)=-I_{b^{-}}^{1-\alpha} \frac{d}{d t} f(t)=-\frac{1}{\Gamma(1-\alpha)} \int_{t}^{b}(\tau-t)^{-\alpha} \frac{d}{d \tau} f(\tau) d \tau .
\end{aligned}
$$

Definition 2.2. [11]. For $m-1<\Re(\mu) \leq m$ and $f \in L^{1}[0, b], 0<t<b \leq \infty$, the left-sided and the right-sided Prabhakar fractional integrals are defined as follows

$$
\begin{aligned}
& \left(\mathbf{E}_{\rho, \mu, \omega, a^{+}}^{\gamma} f\right)(t)=\int_{a}^{t}(t-\tau)^{\mu-1} E_{\rho, \mu}^{\gamma}\left(\omega(t-\tau)^{\rho}\right) f(\tau) d \tau, \\
& \left(\mathbf{E}_{\rho, \mu, \omega, b^{-}}^{\gamma} f\right)(t)=\int_{t}^{b}(\tau-t)^{\mu-1} E_{\rho, \mu}^{\gamma}\left(\omega(\tau-t)^{\rho}\right) f(\tau) d \tau,
\end{aligned}
$$

where $\rho, \mu, \omega, \gamma \in \mathbb{C}$ and $E_{\rho, \mu}^{\gamma}$ is Prabhakar function(1-3).

Definition 2.3. [11]. For the function $f \in L^{1}[0, b]$, the left-sided and the right-sided Prabhakar fractional derivatives are defined as

$$
\begin{aligned}
\left(D_{\rho, \mu, \omega, a^{+}}^{\gamma} f\right)(t) & =\frac{d^{m}}{d t^{m}} \mathbf{E}_{\rho, m-\mu, \omega, a^{+}}^{-\gamma} f(t), \\
\left(D_{\rho, \mu, \omega, b^{-}}^{\gamma} f\right)(t) & =(-1)^{m} \frac{d^{m}}{d t^{m}} \mathbf{E}_{\rho, m-\mu, \omega, b^{-}}^{-\gamma} f(t),
\end{aligned}
$$

where $m-1<\Re(\mu) \leq m$. For the absolutely continuous function $f$, the left-sided and the right-sided Caputo-Prabhakar fractional derivatives are also defined as follows:

$$
\begin{aligned}
& C_{\mathbb{D}_{\rho, \mu, \omega, a^{+}}^{\gamma}}^{\gamma} f(t)=\mathbf{E}_{\rho, m-\mu, \omega, a^{+}}^{-\gamma} \frac{d^{m}}{d t^{m}} f(t), \\
& { }^{C} \mathbb{D}_{\rho, \mu, \omega, b^{-}}^{\gamma} f(t)=(-1)^{m} \mathbf{E}_{\rho, m-\mu, \omega, b^{-}}^{-\gamma} \frac{d^{m}}{d t^{m}} f(t) .
\end{aligned}
$$

Lemma 2.4. Let $\rho, \mu, \gamma, \nu, \sigma, \omega \in \mathbb{C}$ that $\Re(\rho), \Re(\mu), \Re(\nu)>0$. Then we have [13]:

$$
\mathbf{E}_{\rho, \mu, \omega, a^{+}}^{\gamma} \mathbf{E}_{\rho, \nu, \omega, a^{+}}^{\sigma} \psi=\mathbf{E}_{\rho, \mu+\nu, \omega, a^{+}}^{\gamma+\sigma} \psi
$$

where $\psi$ is summable function and $\psi \in L^{1}[a, b]$. In particular, the following equation holds:

$$
\mathbf{E}_{\rho, \mu, \omega, a^{+}}^{\gamma} \mathbf{E}_{\rho, \nu, \omega, a^{+}}^{-\gamma} \psi=I_{a^{+}}^{\mu+\nu} \psi
$$

Lemma 2.5. Let $\rho, \mu, \gamma, \omega \in \mathbb{C}$ that $\Re(\rho), \Re(\mu)>0$. Then [13]:

$$
\int_{0}^{t} z^{\mu-1} E_{\rho, \mu}^{\gamma}\left(\omega z^{\rho}\right) d z=t^{\mu} E_{\rho, \mu+1}^{\gamma}\left(\omega t^{\rho}\right) .
$$




\section{EXISTENCE, UNIQUENESS FOR KDVB-EQUATION WITH FRACTIONAL DERIVATIVE CONTAIN THE}

Prabhakar function in Kernel

In this section, we first show the existence of solutions of the KdVB-equation with fractional derivative contain the Prabhakar function in Kernel and then show the uniqueness of it with a theorem. In order to prove existence of solutions of the equation (1-2), We consider the initial conditions of the equation (1-2) as follows:

$$
u(x, 0)=\mathbf{h}(x) .
$$

For this purpose, using formulas (2-11), (2-14) and taking the integration of (1-2), then we have:

$$
u(x, t)-u(x, 0)=\mathbf{E}_{\rho, \mu, \omega, a^{+}}^{\gamma}\left[\sigma \frac{\partial^{2} u(t, x)}{\partial x^{2}}-2 u \frac{\partial u(t, x)}{\partial x}-\delta \frac{\partial^{3} u(t, x)}{\partial x^{3}}\right]
$$

the equations (3-2) can be written as:

$$
u(x, t)-u(x, 0)=\int_{0}^{t}(t-\tau)^{\mu-1} E_{\rho, \mu}^{\gamma}\left(\omega(t-\tau)^{\rho}\right)\left[\sigma u_{x x}-2 u u_{x}-\delta u_{x x x}\right] d \tau .
$$

We consider $\mathbf{Q}(x, t, u, \sigma, \delta)=\sigma u_{x x}-2 u u_{x}-\delta u_{x x x}$ and we show that the Lipschitz conditions are established for the function $\mathbf{Q}(x, t, u, \sigma, \delta)$ that the function $u$ is bounded. Therefore the following relation is established for bounded functions $u, \bar{u}$ :

$$
\begin{aligned}
& \mathbf{Q}(x, t, u, \sigma, \delta)-\mathbf{Q}(x, t, \bar{u}, \sigma, \delta) \\
& =\left(\sigma u_{x x}-2 u u_{x}-\delta u_{x x x}\right)-\left(\sigma \bar{u}_{x x}-2 \bar{u} \bar{u}_{x}-\delta \bar{u}_{x x x}\right) \\
& =\left(\sigma\left(u_{x x}-\bar{u}_{x x}\right)-2\left(u u_{x}-\bar{u} \bar{u}_{x}\right)-\delta\left(u_{x x x}-\bar{u}_{x x x}\right)\right) .
\end{aligned}
$$

Using the features of the norm, we have:

$$
\begin{aligned}
& \|\mathbf{Q}(x, t, u, \sigma, \delta)-\mathbf{Q}(x, t, \bar{u}, \sigma, \delta)\| \\
& =\left\|\sigma\left(u_{x x}-\bar{u}_{x x}\right)+2\left(\bar{u} \bar{u}_{x}-u u_{x}\right)+\delta\left(\bar{u}_{x x x}-u_{x x x}\right)\right\| \\
& \leq \sigma\left\|u_{x x}-\bar{u}_{x x}\right\|+2\left\|\bar{u} \bar{u}_{x}-u u_{x}\right\|+\delta\left\|\bar{u}_{x x x}-u_{x x x}\right\| \\
& =\sigma\left\|\partial_{x x}(u-\bar{u})\right\|+\left\|\partial_{x}\left(\bar{u}^{2}-u^{2}\right)\right\|+\delta\left\|\partial_{x x x}(\bar{u}-u)\right\| .
\end{aligned}
$$

Due to the fact that the functions $u, \bar{u}$ are bounded, in this case there exists a non-negative constants $k_{0}>0, k_{1}>0$ that the following relationship holds for them:

$$
\|u\| \leq k_{0},\|\bar{u}\| \leq k_{0}
$$


Hence, the partial derivative (of order one) $\partial_{x}$ satisfying in the Lipschitz condition, in this case there exists a non-negative constant $K_{1}>0$ that the following relationship holds:

$$
\begin{aligned}
& \|\mathbf{Q}(x, t, u, \sigma, \delta)-\mathbf{Q}(x, t, \bar{u}, \sigma, \delta)\| \\
& \leq \sigma K_{1}^{2}\|u-\bar{u}\|+K_{1}\left\|\bar{u}^{2}-u^{2}\right\|+\delta K_{1}^{3}\|\bar{u}-u\| \\
& \leq \sigma K_{1}^{2}\|u-\bar{u}\|+K_{1}\|\bar{u}-u\|\|\bar{u}+u\|+\delta K_{1}^{3}\|\bar{u}-u\| \\
& \leq\left[\sigma K_{1}^{2}+2 k_{0} K_{1}+\delta K_{1}^{3}\right]\|u-\bar{u}\| .
\end{aligned}
$$

We consider $K=\sigma K_{1}^{2}+2 k_{0} K_{1}+\delta K_{1}^{3}$, thus obtain:

$$
\|\mathbf{Q}(x, t, u, \sigma, \delta)-\mathbf{Q}(x, t, \bar{u}, \sigma, \delta)\| \leq K\|u-\bar{u}\|
$$

It was shown that the function $\mathbf{Q}$ holds in the Lipschitz condition, it allows us to express the following theorem for uniqueness and continuity of the solution of the KdVB-equation :

Theorem 3.1. If $K t^{\mu} E_{\rho, \mu+1}^{\gamma}\left(\omega t^{\rho}\right)<1$, then the non-linear KdVB-equation (1-2) under the initial conditions (3-1) have an unique solution and that solution is continuous.

Proof. Using the relationship (3-3) and we convert it into a iterative scheme as follows:

$$
\begin{aligned}
& u_{0}(x, t)=u(x, 0) \\
& u_{n}(x, t)=\int_{0}^{t}(t-\tau)^{\mu-1} E_{\rho, \mu}^{\gamma}\left(\omega(t-\tau)^{\rho}\right) \mathbf{Q}\left(x, \tau, u_{n}, \sigma, \delta\right) d \tau .
\end{aligned}
$$

Considering

$$
\bar{u}(x, t)=\lim _{n \rightarrow \infty} u_{n}(x, t),
$$

we are now showing that $\bar{u}(x, t)=u(x, t)$ is a solution to the model and that it is also continuous. For this purpose, we define the function $\mathbf{S}_{n}(x, t)$ as follows:

$$
\mathbf{S}_{n}(x, t)=u_{n}(x, t)-u_{n-1}(x, t)
$$

that relationship (3-11) can be rewritten as follows:

$$
u_{n}(x, t)=\sum_{m=0}^{n} \mathbf{S}_{n}(x, t) .
$$

Thus

$$
\begin{aligned}
& \mathbf{S}_{n}(x, t)=u_{n}(x, t)-u_{n-1}(x, t) \\
& =\int_{0}^{t}(t-\tau)^{\mu-1} E_{\rho, \mu}^{\gamma}\left(\omega(t-\tau)^{\rho}\left[\mathbf{Q}\left(x, \tau, u_{n-1}, \sigma, \delta\right)-\mathbf{Q}\left(x, \tau, u_{n-2}, \sigma, \delta\right)\right] d \tau\right.
\end{aligned}
$$


by getting the norm of Eq. (3-13) and applying relationship (3-8), we have:

$$
\begin{aligned}
& \left\|\mathbf{S}_{n}(x, t)=u_{n}(x, t)-u_{n-1}(x, t)\right\| \\
& =\| \int_{0}^{t}(t-\tau)^{\mu-1} E_{\rho, \mu}^{\gamma}\left(\omega(t-\tau)^{\rho}\left[\mathbf{Q}\left(x, \tau, u_{n-1}, \sigma, \delta\right)-\mathbf{Q}\left(x, \tau, u_{n-2}, \sigma, \delta\right)\right] d \tau \|\right. \\
& \leq \int_{0}^{t}(t-\tau)^{\mu-1} E_{\rho, \mu}^{\gamma}\left(\omega(t-\tau)^{\rho}\left\|\mathbf{Q}\left(x, \tau, u_{n-1}, \sigma, \delta\right)-\mathbf{Q}\left(x, \tau, u_{n-2}, \sigma, \delta\right)\right\| d \tau\right. \\
& K \int_{0}^{t}(t-\tau)^{\mu-1} E_{\rho, \mu}^{\gamma}\left(\omega(t-\tau)^{\rho}\left\|u_{n-1}-u_{n-2}\right\| d \tau,\right.
\end{aligned}
$$

equivalently

$$
\left\|\mathbf{S}_{n}(x, t)\right\| \leq K \int_{0}^{t}(t-\tau)^{\mu-1} E_{\rho, \mu}^{\gamma}\left(\omega(t-\tau)^{\rho}\left\|\mathbf{S}_{n-1}\right\| d \tau .\right.
$$

We use the relationship (2-15) and recursive iterations on the relationship (3-15), we get:

$$
\left\|\mathbf{S}_{n}(x, t)\right\| \leq K\left(t^{\mu} E_{\rho, \mu+1}^{\gamma}\left(\omega t^{\rho}\right)\right)^{n} u(x, 0) .
$$

that completes the proof of existence and and continuity results of a solution. To prove the continuity of the solutions of this KdVB-equation, we have:

$$
\begin{aligned}
& u(x, t)-\int_{0}^{t}(t-\tau)^{\mu-1} E_{\rho, \mu}^{\gamma}\left(\omega(t-\tau)^{\rho}\right) \mathbf{Q}\left(x, \tau, u_{n}, \sigma, \delta\right) d \tau \\
& =R_{n}(x, t)+\int_{0}^{t}(t-\tau)^{\mu-1} E_{\rho, \mu}^{\gamma}\left(\omega(t-\tau)^{\rho}\right)\left[\mathbf{Q}\left(x, \tau, u_{n-1}, \sigma, \delta\right)-\mathbf{Q}(x, \tau, u, \sigma, \delta)\right] d \tau
\end{aligned}
$$

thus we have:

$$
\begin{aligned}
& \left\|u(x, t)-\int_{0}^{t}(t-\tau)^{\mu-1} E_{\rho, \mu}^{\gamma}\left(\omega(t-\tau)^{\rho}\right) \mathbf{Q}\left(x, \tau, u_{n}, \sigma, \delta\right) d \tau\right\| \\
& \leq\left\|\mathbf{S}_{n}\right\|+\theta\left(t^{\mu} E_{\rho, \mu+1}^{\gamma}\left(\omega t^{\rho}\right)\right)\left\|\mathbf{S}_{n-1}\right\| .
\end{aligned}
$$

Considering the initial conditions and taking the limit as $n \rightarrow 0$ we have:

$$
u(x, t)=u(x, 0)+\int_{0}^{t}(t-\tau)^{\mu-1} E_{\rho, \mu}^{\gamma}\left(\omega(t-\tau)^{\rho}\right) \mathbf{Q}\left(x, \tau, u_{n}, \sigma, \delta\right) d \tau .
$$

Assume that $u$ and $\bar{u}$ are two solutions of this KdVB-equation, then to prove the unique solution, we have to show $u=\bar{u}$. Using of the Lipschitz condition for $\mathbf{Q}$, we have:

$$
\|u-\bar{u}\| \leq K\left(t^{\mu} E_{\rho, \mu+1}^{\gamma}\left(\omega t^{\rho}\right)\right)\|u-\bar{u}\|
$$

which is equivalent to

$$
\left[1-K\left(t^{\mu} E_{\rho, \mu+1}^{\gamma}\left(\omega t^{\rho}\right)\right)\right]\|u-\bar{u}\| \leq 0
$$

then $\|u-\bar{u}\|=0$, if $K\left(t^{\mu} E_{\rho, \mu+1}^{\gamma}\left(\omega t^{\rho}\right)\right)<1$ that the proof is complete. 


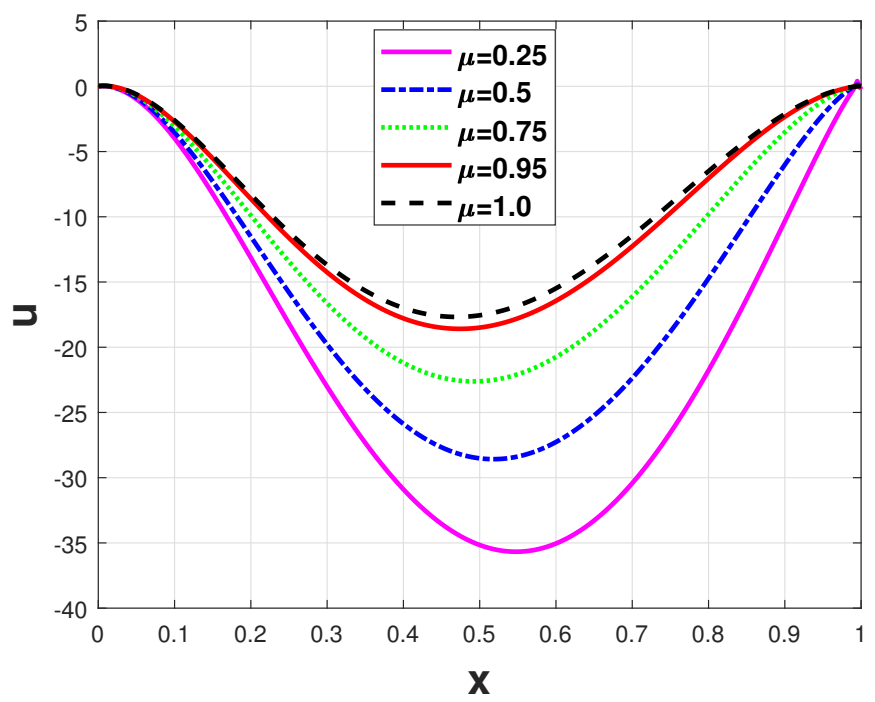

Figure 1. Approximation of solution $u(x, t)$ to system (1-2) for $\mu=$ $0.25,0.5,0.75,0.95,1.0$ and $\rho=1, \gamma=1, \omega=1$.

\section{The numerical simulations approximating the UniQue SOlution to the KdVB-equation}

In this section, we construct a new numerical scheme for KdVB-equation that method is based on the two-step Lagrange polynomial interpolation. For this aim we consider the relation (3-3) and at a given point $t_{n+1}, n=0,1,2, \ldots$, the relation (3-3) is reformulated as:

$$
\begin{aligned}
u\left(x, t_{n+1}\right)-u(x, 0) & =\int_{0}^{t_{n+1}}\left(t_{n+1}-\tau\right)^{\mu-1} E_{\rho, \mu}^{\gamma}\left(\omega\left(t_{n+1}-\tau\right)^{\rho}\right) \mathbf{Q}(x, \tau, u, \sigma, \delta) d \tau \\
& =\sum_{k=0}^{n} \int_{t_{k}}^{t_{n+1}}\left(t_{n+1}-\tau\right)^{\mu-1} E_{\rho, \mu}^{\gamma}\left(\omega\left(t_{n+1}-\tau\right)^{\rho}\right) \mathbf{Q}(x, \tau, u, \sigma, \delta) d \tau
\end{aligned}
$$

using the two-step Lagrange polynomial interpolation for $\mathbf{Q}(x, \tau, u, \sigma, \delta)$ in the interval $\left[t_{k}, t_{n+1}\right]$, thus we have:

$$
\begin{aligned}
P_{k}(\tau) & \left.=\frac{\tau-t_{k-1}}{t_{k}-t_{k-1}} \mathbf{Q}\left(x, t_{k}, u\left(t_{k}\right)\right), \sigma, \delta\right)-\frac{\tau-t_{k}}{t_{k}-t_{k-1}} \mathbf{Q}\left(x, t_{k-1}, u\left(t_{k-1}\right)\right. \\
& =\frac{\mathbf{Q}\left(x, t_{k}, u\left(t_{k}\right), \sigma, \delta\right)}{h}\left(\tau-t_{k-1}\right)-\frac{\mathbf{Q}\left(x, t_{k-1}, u\left(t_{k-1}\right), \sigma, \delta\right)}{h}\left(\tau-t_{k}\right) \\
& \simeq \frac{\mathbf{Q}\left(x, t_{k}, u_{k}, \sigma, \delta\right)}{h}\left(\tau-t_{k-1}\right)-\frac{\mathbf{Q}\left(x, t_{k-1}, u_{k-1}, \sigma, \delta\right)}{h}\left(\tau-t_{k}\right) .
\end{aligned}
$$



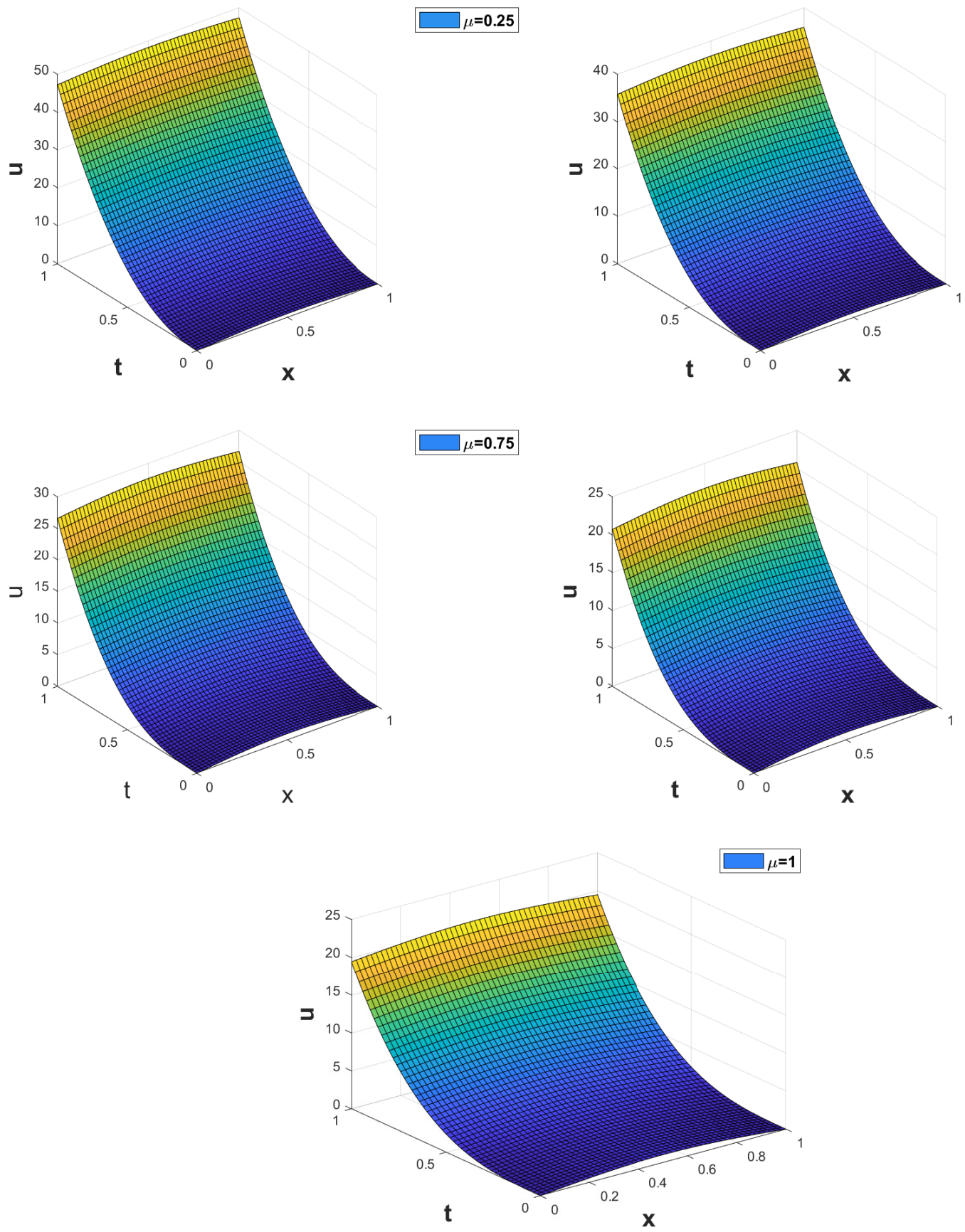

FIGURE 2. Approximation of solution $u(x, t)$ to system (1-2) for $\mu=$ $0.25,0.5,0.75,0.95,1.0$ and $\rho=1, \gamma=1, \omega=1$.

by substituting (4-2) into (4-1) we obtain:

$$
\begin{aligned}
& u_{n+1}=u_{0} \\
& +\sum_{k=0}^{n}\left[\frac{\mathbf{Q}\left(x, t_{k}, u_{k}, \sigma, \delta\right)}{h} \int_{t_{k}}^{t_{k+1}}\left(\tau-t_{k-1}\right)\left(t_{n+1}-\tau\right)^{\mu-1} E_{\rho, \mu}^{\gamma}\left(\omega\left(t_{n+1}-\tau\right)^{\rho}\right) d \tau\right. \\
& \left.-\frac{\mathbf{Q}\left(x, t_{k-1}, u_{k-1}, \sigma, \delta\right)}{h} \int_{t_{k}}^{t_{k+1}}\left(\tau-t_{k}\right)\left(t_{n+1}-\tau\right)^{\mu-1} E_{\rho, \mu}^{\gamma}\left(\omega\left(t_{n+1}-\tau\right)^{\rho}\right) d \tau\right] .
\end{aligned}
$$


Using the relation (1-3) and since the Prabhakar function is convergent [13], thus we obtain:

$$
\begin{aligned}
& u_{n+1}=u_{0} \\
& +\sum_{k=0}^{n} \sum_{m=0}^{\infty} \frac{\omega^{m}(\gamma)_{m}}{m ! \Gamma(\rho m+\mu)}\left[\frac{\mathbf{Q}\left(x, t_{k}, u_{k}, \sigma, \delta\right)}{h} \int_{t_{k}}^{t_{k+1}}\left(\tau-t_{k-1}\right)\left(t_{n+1}-\tau\right)^{\rho m+\mu-1} d \tau\right. \\
& \left.-\frac{\mathbf{Q}\left(x, t_{k-1}, u_{k-1}, \sigma, \delta\right)}{h} \int_{t_{k}}^{t_{k+1}}\left(\tau-t_{k}\right)\left(t_{n+1}-\tau\right)^{\rho m+\mu-1} d \tau\right] .
\end{aligned}
$$

Applying the integration by parts, thus we compute the integral in the relation (4-4)as follows:

$$
\begin{aligned}
& \int_{t_{k}}^{t_{k+1}}\left(\tau-t_{k-1}\right)\left(t_{n+1}-\tau\right)^{\rho m+\mu-1} d \tau=-\left.\left(\tau-t_{k-1}\right) \frac{\left(t_{n+1}-\tau\right)^{\rho m+\mu}}{\rho m+\mu}\right|_{t_{k}} ^{t_{k+1}} \\
& +\int_{t_{k}}^{t_{k+1}} \frac{\left(t_{n+1}-\tau\right)^{\rho m+\mu}}{\rho m+\mu} d \tau .
\end{aligned}
$$

Also with the same process for the second part we obtain:

$$
\begin{aligned}
& \int_{t_{k}}^{t_{k+1}}\left(\tau-t_{k}\right)\left(t_{n+1}-\tau\right)^{\rho m+\mu-1} d \tau=-\left.\left(\tau-t_{k}\right) \frac{\left(t_{n+1}-\tau\right)^{\rho m+\mu}}{\rho m+\mu}\right|_{t_{k}} ^{t_{k+1}} \\
& +\int_{t_{k}}^{t_{k+1}} \frac{\left(t_{n+1}-\tau\right)^{\rho m+\mu}}{\rho m+\mu} d \tau .
\end{aligned}
$$

Substitution relationships (4-5) and (4-6) into (4-4) and using the relation (2-15), thus we get:

$$
\begin{aligned}
& u_{n+1}=u_{0} \\
& +\sum_{k=0}^{n}\left[\frac { \mathbf { Q } ( x , t _ { k } , u _ { k } , \sigma , \delta ) } { h } \left\{-\left.\left(\tau-t_{k-1}\right)\left(t_{n+1}-\tau\right)^{\mu} E_{\rho, \mu+1}^{\gamma}\left(\omega\left(t_{n+1}-\tau\right)^{\rho}\right)\right|_{t_{k}} ^{t_{k+1}}\right.\right. \\
& \left.+\left.\left(t_{n+1}-\tau\right)^{\mu+1} E_{\rho, \mu+2}^{\gamma}\left(\omega\left(t_{n+1}-\tau\right)^{\rho}\right)\right|_{t_{k}} ^{t_{k+1}}\right\} \\
& -\frac{\mathbf{Q}\left(x, t_{k-1}, u_{k-1}, \sigma, \delta\right)}{h}\left\{-\left.\left(\tau-t_{k}\right)\left(t_{n+1}-\tau\right)^{\mu} E_{\rho, \mu+1}^{\gamma}\left(\omega\left(t_{n+1}-\tau\right)^{\rho}\right)\right|_{t_{k}} ^{t_{k+1}}\right. \\
& \left.\left.+\left.\left(t_{n+1}-\tau\right)^{\mu+1} E_{\rho, \mu+2}^{\gamma}\left(\omega\left(t_{n+1}-\tau\right)^{\rho}\right)\right|_{t_{k}} ^{t_{k+1}}\right\}\right] .
\end{aligned}
$$


Thus

$$
\begin{aligned}
& u_{n+1}=u_{0} \\
& +\sum_{k=0}^{n}\left[\frac { \mathbf { Q } ( x , t _ { k } , u _ { k } , \sigma , \delta ) } { h } \left\{-h^{\mu+1}\left[2(n-k)^{\mu} E_{\rho, \mu+1}^{\gamma}\left(\omega h^{\rho}(n-k)^{\rho}\right)\right.\right.\right. \\
& \left.-(n-k)^{\mu+1} E_{\rho, \mu+2}^{\gamma}\left(\omega h^{\rho}(n-k)^{\rho}\right)\right] \\
& \left.+h^{\mu+1}\left[(n-k+1)^{\mu} E_{\rho, \mu+1}^{\gamma}\left(\omega h^{\rho}(n-k+1)^{\rho}\right)-(n-k+1)^{\mu+1} E_{\rho, \mu+2}^{\gamma}\left(\omega h^{\rho}(n-k+1)^{\rho}\right)\right]\right\} \\
& -\frac{\mathbf{Q}\left(x, t_{k-1}, u_{k-1}, \sigma, \delta\right)}{h}\left\{-h^{\mu+1}\left[(n-k)^{\mu} E_{\rho, \mu+1}^{\gamma}\left(\omega h^{\rho}(n-k)^{\rho}\right)\right.\right.
\end{aligned}
$$

$$
\left.\left.-(n-k)^{\mu+1} E_{\rho, \mu+2}^{\gamma}\left(\omega h^{\rho}(n-k)^{\rho}\right)\right]-h^{\mu+1}\left[(n-k+1)^{\mu+1} E_{\rho, \mu+2}^{\gamma}\left(\omega h^{\rho}(n-k+1)^{\rho}\right)\right\}\right] .
$$

Then, we obtained a numerical approximation for the solutions of this KdVB-equation. Now, we consider the present method (4-8) and apply it to solve the model (1-2) under the initial conditions (3-1). we obtain numerical solutions that are represented using $h=0.01$ with the perturbation parameters given by $\sigma=0, \delta=2$ and the initial condition $\mathbf{h}(x)=\sin (\pi x)$ for the different $\mu=0.25, \mu=0.5, \mu=0.75, \mu=0.95, \mu=1.0$ which is shown in the figures 1 and 2 .

\section{REFERENCES}

[1] Z. Artstein, C.W. Gear, I.G. Kevrekidis, M. Slemrod and E.S. Titi, Analysis and computation of a discrete KdV-Burgers type equation with fast dispersion and slow diffusion, SIAM J. Numer. Anal., 49(5)(2011), 2124-2143.

[2] M.H. Derakhshan and A. Ansari, Fractional Sturm-Liouville problems for Weber fractional derivatives, Int. J. Comput. Math., 96(2)(2019), 217-237.

[3] M.H. Derakhshan and A. Ansari, On Hyers-Ulam stability of fractional differential equations with Prabhakar derivatives. Anal., 38(1)(2018), 37-46.

[4] M.H. Derakhshan and A. Ansari, Numerical approximation to Prabhakar fractional Sturm-Liouville problem, J. Comput. Appl. Math., 38(2)(2019).

[5] M.H. Derakhshan, A. Ansari and M. Ahmadi Darani, On asymptotic stability of Weber fractional differential systems, Comput. Methods Differ. Equ., 6(1)(2018), 30-39.

[6] M.A. Firoozjaee and S.A. Yousefi, A numerical approach for fractional partial differential equations by using Ritz approximation, Appl. Math. Comput., 338(2018), 711-721.

[7] E.F.D. Goufo, H.M. Tenkam and M. Khumalo, A behavioral analysis of KdVB equation under the law of Mittag-Leffler function, Chaos. Solitons. Fractals., 125(2019), 139-145.

[8] R. Garra, R. Garrappa, The Prabhakar or three parameter Mittag-Leffler function: Theory and application, Commun. Nonlinear. Sci. Numer. Simul., 56(2018), 314-329. 
[9] R. Garrappa, Numerical solution of fractional differential equations: A survey and a software tutorial, Math., 6(2)(2018), 16.

[10] A. Giusti and I. Colombaro, Prabhakar-like fractional viscoelasticity, Commun. Nonlinear. Sci. Numer. Simul., 56(2018), 138-143.

[11] R. Garra, R. Gorenflo, F. Polito and Ž. Tomovski, Hilfer-Prabhakar derivatives and some applications, Appl. Math. Comput., 242(2014), 576-589.

[12] J. Goodman and P.D. Lax, On dispersive difference schemes, I. Selected Papers Volume I, 41(5)(2005), $545-567$.

[13] A.A. Kilbas, M. Saigo and R.K. Saxena, Generalized Mittag-Leffler function and generalized fractional calculus operators, Integral. Transform. Spec. Funct., 15(1)(2004), 31-49.

[14] D. Kumar and D. Baleanu, Fractional Calculus and its Applications in Physics, Front. Phys., 7(2019), 81.

[15] R.S. Johnson, Shallow water waves on a viscous fluid the undular bore, Phys. Fluids., 15(10) (1972), $1693-$ 1699.

[16] C.H. Su and C.S. Gardner, Korteweg-de Vries equation and generalizations. III. Derivation of the Kortewegde Vries equation and Burgers equation, J. Math. Phys., 10(3) (1969), 536-539.

[17] A.A. Soliman, A numerical simulation and explicit solutions of KdV-Burgers' and Lax's seventh-order KdV equations, Chaos. Solitons. Fractals., 29(2)(2006), 294-302.

[18] I. Podlubny, Fractional differential equations: an introduction to fractional derivatives, fractional differential equations, to methods of their solution and some of their applications (Vol. 198), Elsevier, 1998. 